\title{
Diagnosis and prevention of overtraining syndrome: an opinion on education strategies
}

This article was published in the following Dove Press journal:

Open Access Journal of Sports Medicine

8 September 2016

Number of times this article has been viewed

\author{
Jeffrey B Kreher \\ Department of Orthopaedics, \\ Division of Pediatric Orthopaedics, \\ Massachusetts General Hospital and \\ Massachusetts General Hospital for \\ Children, Boston, MA, USA
}

Correspondence: Jeffrey B Kreher Department of Orthopaedics, Division of Pediatric Orthopaedics, Massachusetts General Hospital and Massachusetts General Hospital for Children, 55 Fruit Street, Suite 3400,

Boston, MA 021।4, USA

$\mathrm{Tel}+\mathrm{I} 6177268523$

Fax + I 617724055 I

Email jkreher@partners.org

\begin{abstract}
Overtraining syndrome is a condition of maladapted physiology in the setting of excessive exercise without adequate rest. The exact etiology and pathogenesis are unknown and being investigated. Symptoms are multisystem in nature and often representative of underlying hormonal, immunologic, neurologic, and psychologic disturbances. Unfortunately, systematic review of the literature does not clearly direct diagnosis, management, or prevention. However, given the severity of symptoms and impairment to quality of life, prevention of overtraining syndrome should be considered by all who interact with endurance athletes. This article will provide suggestions for management of at-risk athletes despite absence of validated diagnostic tests and preventative measures.
\end{abstract}

Keywords: overreaching, unexplained underperformance, burnout, muscle failure syndrome

\section{Introduction}

A condition of overreaching is common in athletes with an incidence ranging from $5 \%$ to $60 \%{ }^{1-7}$ However, overtraining syndrome (OTS) is much less prevalent. ${ }^{8,9}$ Overreaching is a state of excessive volume or intensity of exercise resulting in decreased sport-specific athletic performance. When training loads reach an athlete's individual "tipping point", he or she can be considered overtrained or overreached. With appropriate rest and recovery (and the absence of excessive stress), performance can be increased through supercompensation despite overreaching with the athlete suffering only a temporary performance impairment. ${ }^{8-10}$ OTS is a very specific and severe condition when overtraining without adequate rest and recovery leads to performance decrements that last $>2-3$ months coupled with a mood disturbance. ${ }^{8-11}$ Synonyms used in the literature around overreaching include staleness, burnout, failure adaptation, underrecovery, training stress syndrome, unexplained underperformance syndrome, muscle failure syndrome, and excessive exercise.

OTS is an extreme condition of maladapted physiology. The exact etiology and pathogenesis are unknown and actively being investigated. Symptoms of OTS are multisystem and result from underlying hormonal, immunologic, neurologic, and psychologic disturbances in response to excessive exercise without adequate rest. Endocrine disruptions may be seen in changes to the hypothalamic-pituitary-adrenal and hypothalamicpituitary-gonadal axes. ${ }^{9,12}$ Immune changes with excessive exercise are due to variations in T-cell function and an altered balance between innate and adaptive immunity.,12,13 Neurologic response is through the sympathetic-adrenal-medullary axis. ${ }^{9,12}$ Psychologic 
Table I Terminology of overreaching and overtraining

\begin{tabular}{|c|c|c|c|c|}
\hline Condition & Synonym & Definition & $\begin{array}{l}\text { Performance } \\
\text { decrement }\end{array}$ & Outcome \\
\hline $\begin{array}{l}\text { Functional } \\
\text { overreaching }\end{array}$ & $\begin{array}{l}\text { Short-term } \\
\text { overreaching }\end{array}$ & $\begin{array}{l}\text { Increased training leading to a temporary (day to weeks) performance } \\
\text { decrement and with improved performance after rest }\end{array}$ & Days to weeks & $\begin{array}{l}\text { Positive } \\
\text { (supercompensation) }\end{array}$ \\
\hline $\begin{array}{l}\text { Nonfunctional } \\
\text { overreaching }\end{array}$ & $\begin{array}{l}\text { Long-term } \\
\text { overreaching }\end{array}$ & $\begin{array}{l}\text { Intense training leading to a longer performance decrement (weeks to } \\
\text { months) but with full recovery after rest. Accompanied by increased } \\
\text { psychologic and/or neuroendocrinologic symptoms }\end{array}$ & $\begin{array}{l}\text { Weeks to } \\
\text { months }\end{array}$ & $\begin{array}{l}\text { Negative due to } \\
\text { symptoms and loss of } \\
\text { training time }\end{array}$ \\
\hline $\begin{array}{l}\text { Overtraining } \\
\text { syndrome }\end{array}$ & & $\begin{array}{l}\text { Consistent with extreme nonfunctional OR but with } \\
\text { I) longer performance decrement ( }>2 \text { months) } \\
\text { 2) more severe symptomatology and maladapted physiology } \\
\text { (psychologic, neurologic, endocrinologic, immunologic systems) } \\
\text { 3) accompanied by an additional stressor } \\
\text { 4) not explained by other diseases }\end{array}$ & Months & $\begin{array}{l}\text { Negative due to } \\
\text { symptoms and possible } \\
\text { end to athletic career }\end{array}$ \\
\hline
\end{tabular}

Note: Terms used as defined in Meeusen et $\mathrm{al}^{8}$ and Meeusen et al. ${ }^{9}$

Abbreviation: OR, overreaching.

disruption is believed to be mostly central through changes in neurotransmitter function. ${ }^{9,12}$ It is presumed that the abnormal physiologic states are absent or quickly resolved with rest in the less severe forms of functional overreaching (functional OR) and nonfunctional overreaching (nonfunctional OR). Diagnosis of functional OR, nonfunctional OR, and OTS is complicated by the retrospective nature of their definitions: functional OR is a state of performance decrease lasting days to weeks in the setting of proper rest. Nonfunctional OR shows decreases in performance for weeks to months while OTS is more severe and requires many months for recovery of performance despite rest (Table 1). ${ }^{8,9}$ No single test exists to diagnose OTS and, therefore, exact measures for OTS prevention are unknown. ${ }^{9}$ When athletes are overtrained, there is no validated test to define if they are in a state of functional OR, nonfunctional OR, or OTS. ${ }^{9}$

By definition, OTS is an extreme form of nonfunctional OR with 1) a longer duration of decreased performance for $>2$ months, 2) more severe symptoms and abnormal physiology, and 3) and an additional trigger beyond excessive training load (ie, stressors). ${ }^{8,9,14}$ There is disagreement over whether there is a continuum from nonfunctional OR to OTS ${ }^{11}$ or whether there is a confounding stressor triggering OTS while in functional OR or nonfunctional OR. ${ }^{11,15}$ In short, OTS appears to be a maladapted response to excessive exercise without adequate rest and with perturbations of the hormonal, immunologic, and neurologic systems of the body coupled with altered psychologic function or mood changes. ${ }^{16}$

The reader is directed to a couple of review articles for more in-depth discussion of the physiologic maladaptations of overreached athletes. ${ }^{16,17}$ In addition, the European College of Sport Science and the American College of Sport Medicine have published a joint consensus statement on prevention, diagnosis, and treatment of OTS. ${ }^{9}$ Overreaching has been studied even less in resistance athletes and will not be the focus of this article. This article will cover diagnosis and possible prevention measures for endurance athletes based on presumed physiologic maladaptations in OTS.

\section{Methods}

This is an opinion article supported through a thorough review of the literature. Literature evidence was acquired by a search of relevant articles in English with OVID (1948-2015) and PubMed. Keywords in the search included the following: overtraining syndrome, overtraining, overreaching, unexplained underperformance, staleness, pathophysiology, management, treatment, and evaluation. Finally, bibliographies were reviewed for additional resources. The paper will first cover diagnosis and then prevention of OTS. Each topic will be presented through a review of published literature followed by practical translation and application for the provider and athlete.

\section{Opinion synthesis OTS diagnosis}

\section{Review of the literature}

Diagnosis of OTS would be easy if there was an appropriate biomarker available. Unfortunately, there is no definitive marker able to differentiate functional OR from nonfunctional OR or nonfunctional OR from OTS. While diagnosis of OTS cannot be made definitively with one biomarker, there are a few markers that may be considered in the elite athlete. ${ }^{9,16,18}$ Markers that have been most studied in the literature include the following: urea, uric acid, ammonia, and creatine kinase. ${ }^{18}$ However, one may be able to estimate training load and the body's response with the following: salivary immunoglobulin A, ${ }^{19,20}$ serum testosterone:cortisol, ${ }^{11,18}$ and overnight urinary cortisol:cortisone ratio. ${ }^{21}$ In addition, the 
health care provider must still rule out other medical causes through a thorough history. ${ }^{8,9,16}$ The differential of underperformance includes asthma/bronchial hyperreactivity, thyroid disease, primary mood disorder, adrenal disease, diabetes mellitus or insipidus, iron deficiency with or without anemia, infection, malnutrition, oncologic condition, rheumatologic condition, renal disease, and liver disease among others. ${ }^{16}$ Organic conditions should be screened for in the athlete with persistent underperformance and symptoms of OTS despite a rest period, or immediately if indicated by history. Screening lab tests should include comprehensive metabolic panel, complete blood count, erythrocyte sedimentation rate, C-reactive protein, iron studies, creatine kinase, and thyroid stimulating hormone. A second tier of testing can be added initially or delayed depending on clinical picture and include vitamin B12, folate, serologies for viral hepatitis, toxoplasmosis, cytomegalovirus/Epstein-Barr virus titers, Epstein-Barr virus DNA in saliva, serum, and salivary immunoglobulins (serum immunoglobulin $\mathrm{G}$ subclasses and specific serum immunoglobulin E to aeroallergens), and antinuclear antibodies. ${ }^{16,22}$

While there is no one biomarker for OTS, there are promising biomarkers that remain in the realm of investigation and research. ${ }^{18}$ Due to oxidative stress of excessive exercise, many have investigated the redox status of athletes. Oxidative stress markers such as reduced:oxidized glutathione ratio and urinary isoprostanes are promising for measuring training load and overreached status. ${ }^{23}$ Hematologic markers have been less helpful; however, elevated plasma viscosity has been reported to be specific but not sensitive for nonfunctional OR. ${ }^{24}$ It also makes sense to investigate immunologic markers given the altered immunologic status of athletes with excessive exercise. Increased levels of T-cell activation have been seen in overtrained subjects. ${ }^{25,26}$ Salivary immunoglobulin A levels have shown mixed results with some correlation with upper respiratory infection symptoms and lowered levels in overtrained athletes. ${ }^{20,25,26}$ However, a study in acutely overtrained cyclists found no significant changes. ${ }^{27}$ Finally, given the known disruption in the hypothalamic-pituitary-adrenal axis and hypothalamic-pituitary-gonadal axis, research has been aggressive around hormonal measures. Endocrinologic markers and their studies are quite variable given confounding variables of time of the day, season of the year, phase of menstrual cycle, and nutritional status. ${ }^{8,9,11}$ Two studies have shown value in assessing the training load through testosterone:cortisol ratio $^{11,18}$ and overnight urinary cortisol:cortisone ratio. ${ }^{21}$ However, neither test has been proven to differentiate among functional OR, nonfunctional OR, and OTS.
Since solitary biomarkers are of limited value, the research has also investigated physiologic and/or performance tests to help diagnose OTS. Again, there are tests which help show evidence of excessive load to the body, but they are polluted by inherit and pervasive performance decline that defines the conditions of nonfunctional OR and OTS. ${ }^{28}$ Resting heart, maximal heart rate, and heart rate variabilities do not display ability to differentiate overreaching conditions; however, they do provide a marker for the individual's current physiologic state in relation to recovery from prior work load. ${ }^{18} \mathrm{~A}$ diminished maximal lactate on performance testing does appear specific for nonfunctional OR and OTS but not sensitive to rule out the condition of nonfunctional OR. ${ }^{8,9}$ Recently, a study found discriminate value of combined maximum heart rate and change in lactate concentration from the earlobe during incremental testing after a 3-week protocol of overload training. Athletes labeled as overreached showed a lower maximum heart rate max at lactate threshold and exhaustion compared to a normo-trained population..$^{29}$ If an athlete or coach is going to use field performance tests to screen for overreaching, tests of time to fatigue discriminate better than time trials. ${ }^{11}$

The greatest promise for differentiating functional OR from nonfunctional OR and nonfunctional OR from OTS is a performance test coupled with physiologic markers. Retrospective studies of a two-bout maximal exercise protocol with investigation of the hypothalamic-pituitary-adrenal axis do appear to differentiate functional OR, nonfunctional OR, and OTS. ${ }^{30,31}$ Two bouts of maximal exercise are separated by 4 hours with investigation of adrenocorticotropic hormone and prolactin. Functional OR is believed to occur in an "disturbed state" to excessive exercise with a less pronounced hormonal response to the second bout of exercise. ${ }^{30}$ Nonfunctional OR likely represents the "adapted state" to excessive exercise with an exaggerated hormonal response to the second bout of exercise. ${ }^{30,31}$ OTS is thought to be a "maladapted state" to excessive exercise (usually with an additional stressor as the trigger) where there is an extreme hormonal response to the first bout of exercise and absent hormonal response to the second bout of exercise. It must be noted that the range of total symptom duration for athletes labeled as nonfunctional OR was $\sim 6$ weeks to 8 months and for OTS was 2 years to not fully recovered. The test labeled three athletes as OTS with symptoms for $<6$ months that proceeded to have symptoms after testing and treatment for an additional $3+$ years. In addition, test results of athletes labeled as nonfunctional OR had symptoms for 5 months or less with a time to recovery of 3-6 months after testing. ${ }^{31}$ The results of the two-bout exercise 
Table 2 Summary of symptoms of OTS

\begin{tabular}{|c|c|c|}
\hline $\begin{array}{l}\text { Parasympathetic } \\
\text { alterations } \\
\text { (more common } \\
\text { in aerobic sports) } \\
\end{array}$ & $\begin{array}{l}\text { Sympathetic } \\
\text { alterations } \\
\text { (more common } \\
\text { in anaerobic sports) }\end{array}$ & Other \\
\hline Fatigue & Insomnia & Anorexia \\
\hline Depression & Irritability & Weight loss \\
\hline Bradycardia & Agitation & $\begin{array}{l}\text { Lack of mental } \\
\text { concentration }\end{array}$ \\
\hline \multirow[t]{3}{*}{ Loss of motivation } & Tachycardia & Heavy, sore, stiff muscles \\
\hline & Hypertension & Anxiety \\
\hline & Restlessness & Awakening unrefreshed \\
\hline
\end{tabular}

Notes: Reproduced with permission from Kreher JB, Schwartz JB. Overtraining syndrome: a practical guide. Sports Health. 2012;4(2): 128-138.16

Abbreviation: OTS, overtraining syndrome.

test do question the arbitrary definition of symptoms for 2-3 months despite rest for OTS diagnosis.

\section{Translation to athlete care}

The symptoms of OTS are nonspecific and could be symptoms of many disease states (Table 2). ${ }^{16}$ Medical conditions with symptoms such as fatigue and athletic underperformance include asthma, anemia, hypothyroidism, immunodeficiency, hypocortisolemia, chronic fatigue syndrome, and depression among others. As a result, the diagnosis of functional OR versus nonfunctional OR versus OTS is clinical, retrospective, and only after exclusion of other common conditions. No single diagnostic test or biomarker exists for OTS diagnosis. ${ }^{9,11,16}$ OTS diagnosis is made after a very thorough history of symptoms, nutrition, sleep, injury, and training in reference to the social history, including home, work, and substance use. ${ }^{16}$

Research into the symptoms of overreaching fuels many of the proposed hypotheses to the pathophysiology of OTS. Unfortunately, many of the studies in the name of OTS have been in athletes with functional OR or nonfunctional OR rather than definitive OTS as pointed out in a systematic review. ${ }^{11}$ In addition, research of OTS is limited further by the unethical nature of inducing OTS in subjects given the physiologic and psychologic stresses involved. However, there are two murine models of overreaching both of which have limitations generalizing to human athletes with overreaching. ${ }^{32,33}$ Given these complexities, the more appropriate clinical condition could be labeled unexplained underperformance syndrome during evaluation, which would share many of the preventions measures proposed for OTS. ${ }^{9,16,34}$

While there are no validated or prospective tests to diagnose OTS, an athlete and sports practitioner can and should investigate the body's response to training loads through blood markers and/or physiologic tests. Overreaching may be signaled by an abnormal test in one of the following tests: testosterone:cortisol ration, ${ }^{11,18}$ overnight urinary cortisol:cortisone ratio, ${ }^{21}$ maximal heart rate at lactate threshold, ${ }^{29}$ and/or decreased maximal lactate concentration. ${ }^{9}$ It must be remembered that other, more common, aforementioned medical conditions must also be investigated through history, examination, and blood work. In a study of athletes with persistent fatigue and/or recurrent infections, a cause was found on screening blood work in $68 \%$ with humoral immunodeficiency and unresolved viral infection being the most prevalent at $28 \%$ each. ${ }^{22}$ If no other cause is found in the evaluation of an athlete with severe, prolonged fatigue with performance decrement, one may consider the laborious two-bout exercise test.

\section{OTS prevention}

\section{Review of the literature}

Prevention of overreaching and OTS would involve avoiding stressing the body's homeostasis excessively through exercise. Measures of training load would obviously start with an objective measure. One proposed method is to take the session rate of perceived exertion (RPE) times minutes of workout as a training load. The summation of the session workload over a week or day represents the training load while variance can be analyzed by day or week to give a measure of monotony. The sentinel article found an association between training load (rolling 6-week average), monotony (daily mean divided by standard deviation), and strain (training load times monotony) and minor infections, such as upper respiratory infections. ${ }^{35}$ Many studies in overreaching use the earlier measures of training load, monotony, and strain, thus, giving further support to keeping a training $\log$ with session RPE.

In the past, some have felt low muscle glycogen led to overtraining and OTS given the association of fatigue with inadequate glucose. However, fatigue has not always been correlated with performance decrement ${ }^{36}$ and performance decrement has been seen in those with normal glycogen levels. ${ }^{37}$ Regardless, ensuring adequate fueling of the body is important to avoid excess stress to homeostasis in the setting of excessive exercise.

Fatigue of overtraining has also been blamed on central neurotransmitters given the similarity to fatigue of depression. Metabolism of tryptophan, a precursor to central serotonin, is known to be affected by exercise with increased serum levels, variable uptake centrally due to branched chain amino acid competition, and variable central sensitivity to serotonin. Branched chain amino acids in the bloodstream compete with tryptophan for entry into the central nervous 
system. ${ }^{10,34,38}$ If excess tryptophan entry leads to increased serotonin, fatigue may decrease with branched chain amino acid supplementation as seen in a study of marathon runners. ${ }^{38}$ Well-trained athletes usually are less sensitive to serotonin; however, there is early evidence that favorable adaptation may be lost in OTS..$^{39}$ Therefore, one might show caution with prescribing selective serotonin reuptake inhibitors to overtrained athletes. On the contrary, some understandably encourage aggressive treatment of any mood disturbance, which may even include selective serotonin reuptake inhibitors. ${ }^{40}$

Glutamine has been viewed as a contributor and/or measure of overtraining given research in the immunology realm. Glutamine is an amino acid known to be integral for immune cell function and function in DNA/RNA synthesis, nitrogen transport, gluconeogenesis, and acid-base balance. Studies have found low plasma glutamine after prolonged and repeated high-intensity exercise ${ }^{41,42}$ and in overtrained athletes. ${ }^{34,36} \mathrm{How}$ ever, glutamine supplementation has shown varied responses, including the following: 1) failure to reverse postexercise impairment of immune cells in one study ${ }^{41}{ }^{4}$ ) restoration of macrophage function in overtrained mice, ${ }^{43}$ and 3) possible decrease in rates of early infection among athletes. ${ }^{38,42}$ One may consider supplementation as an attempt at primary or secondary treatment for minor viral infections. However, while many studies note decreased immune function in overreached states, it might be noted that one study has found the incidence of upper respiratory tract infection to be $13 \%$ in overreached athletes and $56 \%$ of non-overreached athletes. ${ }^{44}$

The psychology of overreaching and overtraining has been studied primarily through surveys. Recovery-Stress Questionnaire for Athletes and Profile of Mood States (POMS) have been studied in the literature extensively. Recovery-Stress Questionnaire for Athletes is a 77-item questionnaire while POMS is a 65 -item questionnaire, which limits their widespread implementation. A study did show a decrease in their defined overreached state with decreased loads when signaled by POMS results. ${ }^{45}$ Measures of "fatigue" and "vigor" and "depression" show the greatest movement during overload training in both healthy and overreached athletes. ${ }^{46}$ In addition, some would contend that fatigue is normal with training but loss of vigor is a signal of a maladapted state. Therefore, A POMS energy index measure has been investigated by taking the difference of fatigue and vigor. The POMS energy index has been found to be sensitive to changes in training stress and recovery. ${ }^{47}$ Finally, a smaller, seven-item questionnaire was developed, the Training Distress Scale, and found to outperform POMS in identifying overtrained swimmers. ${ }^{48}$ The Training Distress Scale has not been used in preventative studies to date. Unfortunately, all surveys suffer from response distortion where answers are not a true representation of current physical and/ or psychological state.

Another potential screening tool to assist in prevention of OTS could be tests of psychomotor speed. While many conditions may show impairment in psychomotor speed, if followed, prospective, psychomotor speed could offer predictive value in potentially overreached athletes. To date, tests of psychomotor speed have not proven sensitive or specific but warrant further investigation given the relative ease of implementation. ${ }^{49,50}$

\section{Translation to athlete care}

As we have seen, the diagnosis of OTS is a complicated clinical endeavor. Therefore, much of the prevention of OTS is simply education of risk factors and ways to prevent excessive stress in the setting of excessive exercise without adequate recovery. One must acknowledge that every individual athlete has a different tipping point for functional OR, nonfunctional OR, and OTS, which may be related to an athlete's stress capacity. ${ }^{15,16}$ The goal of OTS prevention is identifying athletes during episodes of functional OR or nonfunctional OR and educating them of their overreached state and risks of the more serious OTS. In addition, much of the evidence in relation to overreaching and OTS prevention is anecdotal and driven by communication among the athlete, coach, and sports medicine provider.

A framework often used to explain overreaching is the General Adaptation Syndrome of Seyle. ${ }^{51}$ Exercise stress can be acute, subacute, or chronic. Subacute and/or chronic exercise stress is met with a hormonal response to counter the disturbance and establish an adapted state. The hormonal response affects the body's responsiveness to exercise (ie, cardiac, pulmonary, and muscular function) through changes in metabolism (fuel utilization), immunology (cytokine response), neurology (catecholamine response), and psychology (central neurotransmitter changes). ${ }^{10,13,14,40}$ Nonfunctional OR or OTS represents a maladapted state or tipping point in the General Adaptation Syndrome of Selye. However, one must remember to rule out the more common medical diseases, such as anemia, asthma/bronchial hyperreactivity, cardiovascular disease, especially in master athletes, mood disorder, and infection. Medical conditions such as anemia, asthma, infection, and thyroid disorders among others occur much more frequently in endurance athletes than OTS. 
Prevention of OTS and overreaching in general are based on years of self-discovery through endurance exercise and the combined insight of athletes, coaches, researchers, and sports medicine professionals. Common sense means to ensure adequate recovery include the following: keeping a training log and measuring easy physiologic markers, such as weight, morning heart rate, and maximal heart rate. Trends of weight can signal hydration status and potentially nutritional status. Morning heart rate may help signal excessive catecholamines, increased sympathetic tone, or loss of parasympathetic tone. Maximal heart rate often is used as a measure of sympathetic and parasympathetic balance. ${ }^{9,18}$ Newer monitors of heart rate variability and sleep may provide additional signs of inadequate recovery but are yet to be validated in studies. Trends in heart rate variability may help on the individual level but have not been proven beneficial for diagnosis in research studies. ${ }^{52,53}$ In order to prevent overreaching and change training load, one must have measures to guide intervention and measure outcomes of the interventions. Another common screening tool to help prevention is repeated measure of a standardized workout, or performance test, such as a $10 \mathrm{~km}$ run or $40 \mathrm{~km}$ bike time trial just prior to rest period. ${ }^{16,18}$ This would include every effort to maintain relatively the same equipment and environment with the only variable being the athlete.

Communication between athlete and coach/sports medicine professional is paramount given the psychological aspects of serious endurance training and potential for overreaching and less likely OTS. Warning historical elements include 1) reports of increased effort with decreased performance and 2) a loss of reported vigor, which is different than a simple fatigued state. ${ }^{9,10,16,46}$ Some athletes may describe it as an increased RPE for the same workload. One must always consider a more pervasive medical cause for unexplained underperformance. However, medical causes or primary mood disorders tend to be present at the onset of a workout whereas overreaching initially comes with the repeated ability to start workouts but inability to finish the workout and/or loss of a finishing kick. ${ }^{10,16}$ Other sources of stress to body hemostasis to be discussed include mood (affected by work, interpersonal, and hormonal status), nutrition (micro- and macronutrient content and quantity), sleep, environment (ambient heat/coolness, safety of training environment), and body (illness/health, pain, workload, alignment).

It is very important that there is a frank conversation around the psychological aspects of dedicated endurance training. Many would contend OTS does not occur without some psychological stressor in the setting of excessive exercise. ${ }^{9,16}$ It seems self-evident that as the training schedule is periodized, the committed athlete should try to periodize life stressors of work and family (seeking to have personal stressors inversely related to training load). In addition, athlete, coach, and sports medicine professional should be comfortable talking about mood and understand that depressed and anxious moods do not equate to mood disorders, but rather are symptoms of excessive exercise that may be adaptive or maladaptive. In addition, sleep disturbances can be a cause or effect of impaired mood, both of which can also be a cause or effect of overreaching. Finally, the sports medicine provider should recognize that the "unexplained" nature, or inexact etiology, of possible OTS may be existentially challenging to the athletes who are generally in control of so many variables and feel mastery over their body.

\section{Conclusion}

OTS is a rare entity in the realm of overreaching in which 1) excessive exercise is not properly matched with recovery and 2) an excessive stressor leads to significant mood disruption in the setting of maladaptive physiology. While many hypotheses exist as to the causative factors, research is still ongoing. Therefore, diagnosis remains clinical and often one of exclusion. Diagnosis of OTS can only be made after proper clinical evaluation and exclusion of the much more common medical conditions of endurance athletes. Common conditions with presentations similar to OTS include asthma, anemia, hypothyroidism, immunodeficiency, hypocortisolemia, chronic fatigue syndrome, and depression among others. A sports medicine provider could consider initial screening blood work for medical conditions ${ }^{16,22}$ with additional tests to assess the body's response to the training load, such as the following: testosterone:cortisol ratio, ${ }^{11,18}$ overnight urinary cortisol:cortisone ratio, ${ }^{21}$ maximal heart rate at lactate threshold, ${ }^{29}$ and/or decreased maximal lactate concentration. ${ }^{9}$

In addition, all athletes and those involved in their care should be aware of common preventative measures to avoid an overreached state in which maladapted physiology may develop. The hallmark of OTS prevention is education of the health care provider, athlete, and coach(es). The most paramount warning sign athletes should be educated about is the caution one should employ when decreasing performances are meet with increased efforts without a return to better performance. ${ }^{9,16}$ Education of athletes should also include other warning signs for overreaching, such as loss of vigor, ${ }^{46,47}$ loss of finishing kick, ${ }^{10}$ and ability to start a workout but quickly see or feel performance decrement. ${ }^{9,10,16}$ Open communication 
between the athlete and coach and/or sports medicine provider is paramount. All stressors need to be shared among the team to avoid excessive training load. When possible, training plans and life stressors should be inversely matched. Standard tools anecdotally successful in OTS prevention include periodization of training (and life stressors), ${ }^{9,10}$ keeping a training $\log$ with RPE and adjusting volume and intensity based on performance, monotony, strain, and mood, ${ }^{25}$ ensuring adequate calories, hydration, and sleep, ${ }^{9,16}$ abstinence of training during periods of high stress in mood, sleep, environment, and body, ${ }^{9,16}$ and utilization of a psychological monitoring through Recovery-Stress Questionnaire for Athletes, POMS, or Training Distress Scale..$^{9,16,45-48}$ Despite the absence of validated diagnostic and preventative tools, a review of the literature and anecdotal evidence/opinion can be applied to minimize the risk of OTS in endurance athletes.

\section{Disclosure}

The author reports no conflicts of interest in this work.

\section{References}

1. Hooper S, MacKinnon LT, Hanrahan S. Mood states as an indication of staleness and recovery. Int J Sport Psychol. 1997;28:1-12.

2. Koutedakis $Y$, Sharp NC. Seasonal variations of injury and overtraining in elite athletes. Clin J Sport Med. 1998;8(1):18-21.

3. Matos NF, Winsley RJ, Williams CA. Prevalence of non-functional overreaching/overtraining in young English athletes. Med Sci Sports Exerc. 2011:43(7):1287-1294.

4. Morgan WP, Brown DR, Raglin JS, O'Connor PJ, Ellickson KA. Psychological monitoring of overtraining and staleness. Br J Sports Med. 1987;21(3):107-114.

5. Morgan WP, O’Connor PJ, Sparling PB, Pate RR. Psychological characterizations of the elite female distance runner. Int J Sports Med. 1987;8(Suppl 2):124-131.

6. O'Connor PJ, Morgan WP, Raglin JS, Barksdale CM, Kalin NH. Mood state and salivary cortisol levels following overtraining in female swimmers. Psychoneuroendocrinology. 1989;14(4):303-310:462-466.

7. Raglin J, Sawamura S, Alexiou S, Hassmen P, Kentta G. Training practice and staleness in 13-18-year-old swimmers: a cross-cultural study. Ped Exerc Sci. 2000;12(1):61-70.

8. Meeusen R, Duclos M, Gleeson M, Rietjens G, Steinacker J, Urhausen A. Prevention, diagnosis and treatment of the overtraining syndrome: ECSS Position Statement “Task Force.” Eur J Sport Sci. 2006;6(1):1-14.

9. Meeusen R, Duclos M, Foster C, et al. Prevention, diagnosis, and treatment of the overtraining syndrome: joint consensus statement of the European College of Sport Science and the American College of Sports Medicine. Med Sci Sports. Exerc. 2013;45(1):186-205.

10. Budgett R. Fatigue and underperformance in athletes: the overtraining syndrome. Br J Sports Med. 1998;32(2):107-110.

11. Halson SL, Jeukendrup AE. Does overtraining exist? An analysis of overreaching and overtraining research. Sports Med. 2004;34(14):967-981.

12. Fry RW, Morton AR, Keast D. Overtraining in athletes: an update. Sports Med. 1991;12(1):32-65.

13. Lakier Smith L. Overtraining, excessive exercise, and altered immunity: is this a T helper-1 versus T helper-2 lymphocyte response? Sports Med. 2003;33(5):347-364.

14. Robson P. Elucidating the unexplained underperformance syndrome in endurance athletes: the interleukin-6 hypothesis. Sports Med. 2003;33(10):771-781.
15. Kenttä G, Hassmén P. Overtraining and recovery: a conceptual model. Sports Med. 1998;26(1):1-16.

16. Kreher JB, Schwartz JB. Overtraining syndrome: a practical guide. Sports Health. 2012;4(2):128-138.

17. Carfagno DG, Hendrix JC 3rd. Overtraining syndrome in the athlete: current clinical practice. Curr Sports Med Rep. 2014; 13(1):45-51.

18. Urhausen A, Kindermann W. Diagnosis of overtraining - what tools do we have? Sports Med. 2002:32;95-102.

19. Mackinnon LT. Chronic exercise training effects on immune function. Med Sci Sports Exerc. 2000;32(Suppl 7):S369-S376.

20. Mackinnon LT, Hooper SL. Plasma glutamine and upper respiratory tract infection during intensified training in swimmers. Med Sci Sports Exerc. 1996;28(3):285-290.

21. Gouarné C, Groussard C, Gratas-Delamarche A, Delamarche P, Duclos M. Overnight urinary cortisol and cortisone add new insights into adaptation to training. Med Sci Sports Exerc. 2005;37(7):1157-1167.

22. Reid VL, Gleeson M, Williams N, Clancy RL. Clinical investigation of athletes with persistent fatigue and/or recurrent infections. Br J Sports Med. 2004;38(1):42-45.

23. Margonis K, Fatouros IG, Jamurtas AZ, et al. Oxidative stress biomarkers responses to physical overtraining: implications for diagnosis. Free Radic Biol Med. 2007;43(6):901-910.

24. Varelet-Marie E, Mercier J, Brun JF. Is plasma viscosity a predictor of overtraining in athletes? Clin Hemorheol Microcirc. 2006;35(1-2): 329-332.

25. Fry RW, Grove JR, Morton AR, Zeroni PM, Gaudieri S, Keast D. Psychological and immunological correlates of acute overtraining. $\mathrm{Br}$ J Sports Med. 1994;28(4):241-246.

26. Gabriel H, Kindermann W. The acute immune response to exercise: what does it mean? Int J Sports Med. 1997;18(Suppl 1):S28-S45.

27. Halson SL, Lancaster GI, Jeukendrup AE, Gleeson M. Immunological responses to overreaching in cyclists. Med Sci Sports Exerc. 2003;35(5):854-861.

28. Meeusen R, Duclos M, Gleeson M, Rietjens G, Steinacker J, Urhausen A. The overtraining syndrome - facts and fiction. Eur J Sport Sci. 2006;6(4):263.

29. Le Meur Y, Hausswirth C, Natta F, Couturier A, Bignet F, Vidal PP. A multidisciplinary approach to overreaching detection in endurance trained athletes. J Appl Physiol. 2013;114(3):411-420.

30. Meeusen R, Piacentini MF, Busschaert B, Buyse L, De Schutter G, Stray-Gundersen J. Hormonal response in athletes: the use of a two bout exercise protocol to detect subtle differences in (over)training status. Eur J Appl Physiol. 2004;91(2-3):140-146.

31. Meeusen R, Nederhof E, Buyse L, Roelands B, deSchutter G, Piacentini MF. Diagnosing overtraining in athletes using the two-bout exercise protocol. Br J Sports Med. 2010;44:642-648.

32. Hohl R, Ferraresso RL, De Oliveira RB, Lucco R, Brenzikofer R, De Macedo DV. Development and characterization of an overtraining animal model. Med Sci Sports Exerc. 2009;41(5):1155-1163.

33. Pereira BC, Filho LA, Alves GF, et al. A new overtraining protocol for mice based on downhill running sessions. Clin Exp Pharmacol Physiol. 2012;39(9):793-798.

34. Budgett R, Newsholme E, Lehmann M, et al. Redefining the overtraining syndrome as the unexplained underperformance syndrome. Br J Sports Med. 2000;34(1):67-68.

35. Foster C. Monitoring training in athletes with reference to overtraining syndrome. Med Sci Sports Exerc. 1998;30(7):1164-1168.

36. Costill DL, Flynn MG, Kirwan JP, et al. Effects of repeated days of intensified training on muscle glycogen and swimming performance. Med Sci Sports Exerc. 1988;20:249-254.

37. Snyder AC, Kuipers H, Cheng B, Servais R, Fransen E. Overtraining following intensified training with normal muscle glycogen. Med Sci Sports Exerc. 1995;27(7):1063-1070.

38. Castell LM, Poortmans JR, Leclercq R, Brasseur M, Duchateau J, Newsholme EA. Some aspects of the acute phase response after a marathon race, and the effects of glutamine supplementation. Eur J Appl Physiol. 1997;75:47-53. 
39. Budgett R, Hiscock N, Arida R, Castell LM. The effects of the 5-HT2C agonist m-chlorphenylpiperazine on elite athletes with unexplained underperformance syndrome (overtraining). Br J Sports Med. 2010;44:280-283.

40. Armstrong LE, VanHeest JL. The unknown mechanism of the overtraining syndrome: clues from depression and psychoneuroimmunology. Sports Med. 2002;32:185-209.

41. Hiscock N, Pedersen BK. Exercise-induced immunosuppression-plasma glutamine is not the link. J Appl Physiol. 2002;93:813-822.

42. Walsh NP, Blannin AK, Robson PJ, Gleeson M. Glutamine, exercise and immune function. Sports Med. 1998;28(3):177-191.

43. Xiao W, Chen P, Dong J, Wang R, Luo B. Dietary glutamine supplementation partly reverses impaired macrophage function resulting from overload training in rats. Int J Sport Nutr Exerc Metab. 2015;25(2): 179-187.

44. Mackinnon LT, Hooper SL. Mucosal (secretory) immune system responses to exercise of varying intensity and during overtraining. Int J Sports Med. 1994;15 (Suppl 3):S179-S183.

45. Berglund B, Safstrom H. Psychological monitoring and modulation of training load of world-class canoeists. Med Sci Sports Exerc. 1994;26:1036-1040.
46. Gustafsson H, Holmberg H, Hassmen P. An elite endurance athlete's recovery from underperformance aided by a multidisciplinary sport science support team. Eur J Sport Sci. 2008;8(5):267-276.

47. Kentta G, Hassmen P, Raglin J. Overtraining and staleness in Swedish age-group athletes: association with training behavior and psychosocial stressors. Int J Sports Med. 2001;22:460-465.

48. Raglin J, Morgan W. Development of a scale for use in monitoring training-induced distress in athletes. Int J Sports Med. 1994;15:84-88.

49. Nederhof E, Lemmeink K, Visscher C, Meeusen R, Mulder T. Psychomotor speed, possibly a new marker for overtraining syndrome. Sports Med. 2006;36:817-828.

50. Nederhof E, Lemmink K, Zwerver J, Mulder T. The effect of high load training on psychomotor speed. Int J Sports Med. 2007;28:595-601.

51. Selye H. Syndrome produced by diverse nocuous agents. Nature. 1936;138:32.

52. Hynynen A, Uusitalo A, Konttinen N, Rusko H. Heart rate variability during night sleep and after awakening in overtrained athletes. Med Sci Sports Exerc. 2006;38(2):313-317.

53. Pichot V, Roche F, Gaspoz JM. Relation between heart rate variability and training load in middle-distance runners. Med Sci Sports Exerc. 2000;32:1729-1736.
Open Access Journal of Sports Medicine

\section{Publish your work in this journal}

The Open Access Journal of Sports Medicine is an international, peer-reviewed, open access journal publishing original research, reports, reviews and commentaries on all areas of sports medicine. The journal is included on PubMed. The manuscript management system is completely online and includes a very quick and fair
Dovepress

peer-review system. Visit http://www.dovepress.com/testimonials.php to read real quotes from published authors. 